\title{
A MODULI SPACE OF EXOTIC $\mathbb{R}^{4}$ 's
}

\author{
by ROBERT E. GOMPF*
}

(Received 6th March 1988)

1.

In 1982 , the first exotic $\mathbb{R}^{4}$ was discovered-a smooth manifold homeomorphic to $\mathbb{R}^{4}$, but not diffeomorphic to it. The object shocked topologists by its open defiance of the rules of high-dimensional smoothing theory. The exotic $\mathbb{R}^{4}$ was constructed by connecting the two powerful machines of Freedman [4] and Donaldson [2] to earlier work of Casson [1].

More recently, many other examples have been discovered. Freedman and Taylor [5] have constructed a "universal" $\mathbb{R}^{4}$ in which all others embed. A continuous family of distinct exotic $\mathbb{R}^{4}$ 's has been constructed $[6,7]$, which is naturally parametrized by the two-dimensional space $I \times I$. (We take $I$ to be the closed interval $[1, \infty]$. See [6, Theorem 3.1] and the last paragraph of $[6, \S 3]$.) These examples are "large" in the sense that they contain compact 4-manifolds which cannot embed in any homotopy 4-sphere. In contrast, there is now known to be an exotic $\mathbb{R}^{4}$ which embeds in the standard $\mathbb{R}^{4}$. (This follows from work of Casson ([1, Lecture III]) together with Donaldson's counterexample to the smooth $h$-cobordism theorem [3] and Freedman theory.)

A natural question is how to organize the set of exotic $\mathbb{R}^{4}$ 's. We let $\mathscr{R}$ denote the set of all oriented diffeomorphism types homeomorphic to $\mathbb{R}^{4} . \mathscr{R}$ contains a 2-parameter subspace, and it seems a good conjecture that there are many other parameters which are presently hidden. Such a space cries out for a topology. In the present paper we construct a quotient space $\mathscr{R}_{\sim}$, modding out by an equivalence relation which, for example, identifies with $\mathbb{R}^{4}$ any exotic $\mathbb{R}^{4}$ embedding inside it. With this exception, all presently known structure of $\mathscr{R}$ is preserved in $\mathscr{R}_{\sim}$. We endow the space $\mathscr{R}_{\sim}$ with a topology which is metrizable and has a countable basis. In particular, this cannot be the discrete topology, since $\mathscr{R}_{\sim}$ is uncountable. The topology has some compatibility with the monoid structure on $\mathscr{R}_{\sim}$ induced by end-sum (see $\S 3$ ), and is a refinement of the partial order topology induced by "ミ" (see $\S 2$ ) which is essentially inclusion. $\mathscr{R}_{\sim}$ also has a "one-sided" compactness property: every increasing sequence converges.

The moduli space $\mathscr{R}_{2}$ is somewhat analogous to moduli spaces of complex structures. One quirk of the latter is that deformation spaces need not be Hausdorff. This is corrected by removing certain "degenerate" elements from the space. In the case of $\mathscr{R}_{\sim}$ a

* Supported by a National Science Foundation postdoctoral fellowship. 
similar phenomenon can occur, but it is too pervasive to be corrected in this manner. Instead, we have forced the topology to be Hausdorff, at the expense of having natural 1-parameter families which are not continuous, but compatible with part of the topology of $\mathscr{R}_{\sim}$ (see $\S 3$ ). In fact, the above embedding of $I \times I$ is not known to be continuous, but it is compatible with part of the topology.

In this article, we work entirely in the smooth category except when otherwise stated. All manifolds are implicitly oriented, and all embeddings are assumed to be smooth and orientation-preserving.

2.

We would like to partially order $\mathscr{R}$ by inclusion. To avoid technical problems involving noncompactness, we adopt the following definition.

Definition 2.1. Given $R_{1}, R_{2} \in \mathscr{R}$, we say $R_{1} \leqq R_{2}$ if any oriented compact 4-manifold (with boundary) which embeds in $R_{1}$ also embeds in $R_{2}$.

This is clearly reflexive and transitive. It generalizes inclusion in the sense that $R_{1} \leqq R_{2}$ whenever $R_{1}$ embeds in $R_{2}$.

The above relation is not a partial ordering on $\mathscr{R}$. In particular, any exotic $\mathbb{R}^{4}$ which embeds in the standard one is both $\geqq \mathbb{R}^{4}$ and $\leqq \mathbb{R}^{4}$. We remedy this as follows:

Definition 2.2. $R_{1}$ and $R_{2}$ are compactly equivalent, $R_{1} \sim R_{2}$, if both $R_{1} \leqq R_{2}$ and $R_{2} \leqq R_{1}$.

Roughly speaking, $R_{1}$ and $R_{2}$ are compactly equivalent if we cannot distinguish them by looking at compact subsets. Let $\mathscr{R}_{\sim}$ denote the space of compact equivalence classes in $\mathscr{R}$. Then $\mathscr{R}_{\sim}$ is partially ordered by $\leqq$. The 2-parameter family of [6] is embedded in $\mathscr{R}_{\sim}$. (See the remark following Theorem 3.1 of [6].) In fact, the embedding sends $I \times I$ isomorphically onto its image as a map of partially ordered spaces. (Here, $I \times I$ is ordered by $\left(a_{1}, b_{1}\right) \leqq\left(a_{2}, b_{2}\right)$ if and only if $a_{1} \leqq a_{2}$ and $b_{1} \leqq b_{2}$.)

As a first attempt at topologizing $\mathscr{R}_{\sim}$ we can consider the partial order topology. A subbasis of closed sets is given by $K_{R}, L_{R}$ indexed by $R \in \mathscr{R}_{\sim}$, where $K_{R}=\left\{R^{\prime} \in \mathscr{R}_{\sim} \mid R^{\prime} \leqq\right.$ $R\}$ and $L_{R}=\left\{R^{\prime} \in \mathscr{R}_{-} \mid R \leqq R^{\prime}\right\}$. Unfortunately, this topology is hard to work with, and not even known to be Hausdorff.

Our topology $\tau$ for $\mathscr{R}_{\sim}$ is a refinement of the partial order topology. We consider sets $U_{X}$ indexed by compact, oriented 4-manifolds $X . U_{X}=\left\{R \in \mathscr{R}_{\sim} \mid X\right.$ embeds in $\left.R\right\}$. These will be open sets in $\tau$. In fact, we define $\tau$ by the subbasis of closed sets $\mathscr{R}_{-}-U_{X}$ and $L_{R}$. It is routine to verify that the sets $K_{R}$ are closed in this topology, so that $\tau$ is indeed a refinement of (possibly equal to) the partial order topology.

Proposition 2.3. The topology $\tau$ is regular.

Proof. The key idea is the following: Let $X$ be a compact 4-manifold embedded in some $R \in \mathscr{R}$. Then there is a compact $X^{\prime}$ and some $R^{\prime} \in \mathscr{R}$ such that $X \subset R^{\prime} \subset X^{\prime} \subset R$. To 
see this, recall that $R$ is homeomorphic to $\mathbb{R}^{4}$. Thus, $X$ lies in the interior of some compact topological 4-ball $B$. We may take $R^{\prime}=$ int $B$ with the smooth structure inherited from $R$, and $X^{\prime}=f^{-1}[0,1]$ where $f: R \rightarrow[0, \infty)$ is a smooth, proper map which vanishes on $B$ and takes 1 as a regular value.

It is now easy to prove regularity. $\tau$ is $T_{1}$, since the one-point set $\{R\}=K_{R} \cap L_{R}$ is closed. Given a closed set of the form $\mathscr{R}_{-}-U_{X}$, and $R$ not in this set, we must have $X \subset R$, so we can write $X \subset R^{\prime} \subset X^{\prime} \subset R$ as above. The disjoint open sets $U_{X^{\prime}}$ and $\mathscr{R}_{\sim}-L_{R^{\prime}}$ contain $R$ and $\mathscr{R}_{\sim}-U_{X}$, respectively. Similarly, to separate a closed set $L_{R}$ from a point $R^{*} \notin L_{R}$ observe that by definition there is a compact $X \subset R$ which does not embed in $R^{*}$. Writing $X \subset R^{\prime} \subset X^{\prime} \subset R$, we obtain the desired open sets $\mathscr{R}_{\sim}-L_{R^{\prime}}$ and $U_{X^{\prime}}$, containing $R^{*}$ and $L_{R}$. It is now trivial to separate points from arbitrary closed sets.

Proposition 2.4. The topology $\tau$ has a countable basis. Hence, it is metrizable.

Proof. The second statement follows from the first, together with Proposition 2.3, via the Urysohn Metrization Theorem.

There are only countably many compact manifolds, since there are only countably many finite simplicial complexes. Thus, there are only countably many sets of the form $U_{X}$, and it suffices to show that the sets $L_{R}$ are generated by a countable subcollection.

Consider all pairs $\left(X^{\prime}, X\right)$ of compact 4-manifolds for which there is an $R \in \mathscr{R}$ with $X \subset R \subset X^{\prime}$. For each such pair, arbitrarily choose one such $R$ and denote it $R\left(X^{\prime}, X\right)$. This gives a countable collection of elements $R\left(X^{\prime}, X\right) \in \mathscr{R}$. To see that the closed sets $L_{R\left(X^{\prime}, X\right)}$ generate, choose a set $L_{R}$ and an $R^{*} \notin L_{R}$. As above, we have an $X$ not embedding in $R^{*}$ with $X \subset R^{\prime} \subset X^{\prime} \subset R$. Thus there is an element $R\left(X^{\prime}, X\right)$, and we have $R^{*} \notin L_{R\left(X^{*}, X\right)}$ and $L_{R} \subset L_{R\left(X^{\prime}, X\right)}$.

\section{Proposition 2.5. Every increasing sequence converges.}

Proof. Given an increasing sequence $R_{1} \leqq R_{2} \leqq R_{3} \leqq \ldots$ in $\mathscr{R}$ we will construct an $R$ for which (1) each $R_{n} \leqq R$ and (2) any compact $X \subset R$ embeds in some $R_{n}$. It is then easy to verify that $R_{n} \rightarrow R$ in the topology $\tau$. (Note that if $R_{1} \subset R_{2} \subset R_{3} \subset \ldots$ then $R=\bigcup_{n=1}^{\infty} R_{n}$ suffices.)

For each $n$, let $\left\{X_{n, i} \mid i=1,2,3, \ldots\right\}$ be an increasing sequence of compact submanifolds whose union is $R_{n}$. Then $X_{n, i}$ embeds in $R_{m}$ for $m \geqq n$. Inductively define compact manifolds $Y_{m} \subset R_{m}$ as follows: Let $Y_{1}=X_{1,1} \subset R_{1}$. Now assume $Y_{m} \subset R_{m}$ has been defined. Fix embeddings (possibly overlapping) of $Y_{m}, X_{m, 1}, X_{m-1,2}, \ldots, X_{1, m}$ in $R_{m+1}$. Let $B_{m+1} \subset R_{m+1}$ be a topological 4-ball whose interior contains the images of all of these manifolds. Let $Y_{m+1}$ be a compact (smooth) manifold in $R_{m+1}$ containing $B_{m+1}$. By construction, we now have embeddings $Y_{1} \subset Y_{2} \subset Y_{3} \subset \ldots$. Let $R=\bigcup_{m=1}^{\infty} Y_{m}$. Since each embedding factors $Y_{m} \subset B_{m+1} \subset Y_{m+1}, R$ is topologically a nested union of 4-balls, so it is homeomorphic to $\mathbb{R}^{4}$. Condition (1) holds since any compact subset of $R_{n}$ lies in some $X_{n, i} \subset Y_{n+i}$, and condition (2) is clear. 
3.

Our subbasis for $\tau$ naturally divides into two parts. The sets $U_{X}$ are natural in many ways, but the sets $L_{R}$ are harder to deal with. For example, the embedding $I \times I \rightarrow \mathscr{R}_{-}$of [6] is easily seen to be $U$-continuous, i.e., continuous with respect to the (non- $T_{1}$ ) topology generated by the sets $U_{X}$ alone. It is not clear, however, that the preimage of $L_{R}$ will be closed for $R$ not in the family.

On the other hand, the most general parametrized families should not always be continuous. We may obtain a 1-parameter family from any sliced concordance. This is a smoothing on $\mathbb{R}^{4} \times I$ such that projection $\pi$ onto $I$ is a smooth submersion. The 1-parameter family is given by $R_{t}=\pi^{-1}(t), t \in I$. A sliced concordance is analogous to a deformation of complex structures. Any sliced concordance is $U$-continuous. (If a compact $X$ embeds in $R_{t_{0}}$ then it embeds in $R_{t}$ for $t$ near $t_{0}$.) However, the following example cannot be continuous in any Hausdorff topology: Let $R \subset R^{\prime}$ be an arbitrary nested pair for which $R$ is the interior of a flat topological 4-ball in $R^{\prime}$. A sliced concordance may be obtained from $R^{\prime} \times I$ by removing all of the bottom boundary except for $R \times\{0\}$. Then $R_{0}=R$ and $R_{t}=R^{\prime}$ for $0<t \leqq 1$. Similar examples occur with complex structures. Since $R$ and $R^{\prime}$ are nearly arbitrary, however, it seems impractical to eliminate this phenomenon by throwing away part of the space $\mathscr{R}_{\sim}$.

The space $\mathscr{R}$ is a commutative monoid under the operation of end-sum, $\bar{q}$, the noncompact analogue of boundary-sum. (See the appendix of [6] for the defintion and basic properties.) End-sum is easily seen to be compatible with $\leqq .\left(R_{1} \leqq R_{2}\right.$ and $R_{3} \leqq R_{4} \Rightarrow R_{1} \bigsqcup R_{3} \leqq R_{2} \bigsqcup R_{4}$. This is one reason why we restricted to compact sets when defining $\leqq$.) Thus, $\mathscr{R}_{\sim}$ inherits a monoid structure. End-sum is easily seen to be jointly $U$-continuous on $\mathscr{R}_{\sim}$, but again the sets $L_{R}$ cause trouble.

The space $\mathscr{R}_{\sim}$ contains unique maximal and minimal elements. Clearly, $\mathbb{R}^{4} \leqq R$ for any $R \in \mathscr{R}$, and this characterizes $\mathbb{R}^{4}$ up to compact equivalence. We may also construct an element $R^{*}$ characterized up to compact equivalence by the property that $R \leqq R^{*}$ for any $R \in \mathscr{R}$. To construct $R^{*}$, consider all compact 4-manifolds $X$ which embed in elements of $\mathscr{R}$. For each $X$, choose an element $R_{X} \in \mathscr{R}$ in which $X$ embeds. This gives a countable collection of elements $R_{X}$. Let $R^{*}$ be the end-sum of all of these. Then for any $R \in \mathscr{R}$, all compact subsets of $R$ embed in $R^{*}$, as required. This $R^{*}$ is actually diffeomorphic to the universal $\mathbb{R}^{4}$ of Freedman and Taylor [5]. (In fact, our collection of $X$ 's includes Freedman and Taylor's "link slice solutions" infinitely many times, which characterizes the universal $\mathbb{R}^{4}$.)

The techniques of this paper can be applied to the study of smooth structures on other open 4-manifolds. For example, let $\mathscr{S}_{n}$ denote the space of smoothings on a 4-sphere where $n$ punctures, up to orientation and end-preserving diffeomorphism. Of particular interest are $\mathscr{S}_{1}=\mathscr{R}$ and $\mathscr{S}_{2}=$ the space of smoothings on $S^{3} \times \mathbb{R}$. Section 2 generalizes directly. We simply restrict attention to compact, oriented 4-manifolds $X$ which have exactly $n$ boundary components, indexed by $\{1, \ldots, n\}$. Embeddings $X \rightarrow$ $S \in \mathscr{S}_{n}$ are required to match the ith boundary of $X$ with the $i$ th end of $S$. Note that the spaces $\mathscr{S}_{n}$ - will be disconnected for $n \geqq 2$ since the Kirby-Siebenmann invariant gives a continuous surjection $\mathscr{S}_{n \sim} \rightarrow H^{3}\left(S^{4}-n\right.$ points; $\left.\mathbb{Z}_{2}\right)$. In this setting, we have additional 
structures: inclusion i: $\mathscr{S}_{n} \rightarrow \mathscr{S}_{n+1}$ by deleting a point, projections $r: \mathscr{S}_{n+1} \rightarrow \mathscr{S}_{n}$ by coalescing two preassigned ends (i.e., deleting a smooth curve connecting the ends) and connected sum \#: $\mathscr{S}_{m} \times \mathscr{S}_{n} \rightarrow \mathscr{S}_{m+n}$. These operations all descend to $\mathscr{S}_{n \sim}$ and are $U$-continuous. Note that for $\mathscr{S}_{1}=\mathscr{R}, R_{1} \square R_{2}=r\left(R_{1} \# R_{2}\right)$. A similar monoid structure exists on each $\mathscr{S}_{n}$.

\section{REFERENCES}

1. A. CASSON, Three lectures on new constructions in 4-dimensional manifolds, notes prepared by L. Guillou, Prépublications Orsay 81 T06.

2. S. Donaldson, An application of gauge theory to four-dimensional topology, J. Differential Geom. 18 (1983), 279-315.

3. S. Donaldson, La topologie différentielle des surfaces complexes, C.R. Acad. Sci. 301 (1985), 317-320.

4. M. H. Freedman, The topology of four-dimensional manifolds, J. Differential Geom. 17 (1982), 357-453.

5. M. H. Freedman and L. TAYLoR, A universal smoothing of four-space, J. Differential Geom. 24 (1986), 69-78.

6. R. GompF, An infinite set of exotic $\mathbb{R}^{4}$ 's, J. Differential Geom. 21 (1985), 283-300.

7. C. TAuBes, Gauge theory on asymptotically period 4-manifolds, J. Differential Geom. 25 (1987), 363-430.

The UNIVERSITY OF TeXAS

Austin, TX 78712, USA 